\title{
Hands on GERDA: Energy calibration and resolution of a BEGe detector with a ${ }^{228}$ Th source
}

\author{
Dana Byram* ${ }^{\dagger}$ \\ University of South Dakota \\ Sanford Underground Research Facility \\ E-mail: dbyramesanfordlab.org

\section{Carla Macolino} \\ Gran Sasso Science Institute and \\ Laboratori Nazionali del Gran Sasso, INFN L'Aquila ITALY \\ E-mail: carla.macolino@lngs.infn.it
}

\begin{abstract}
In this paper we report the results of the hands on experimental activity performed at Laboratori Nazionali del Gran Sasso during the Gran Sasso Summer Institute 2014. The research activity consisted in the energy calibration and resolution measurement of a Broad Energy Germanium detector within the GERDA experiment for the search for neutrinoless double beta decay.

The procedure to retrieve the calibration curve and the estimation of the energy resolution in the region around the Q-value are discussed.
\end{abstract}

Gran Sasso Summer Institute 2014 Hands-On Experimental Underground Physics at LNGS 22 September - 03 October 2014

INFN - Laboratori Nazionali del Gran Sasso, Assergi, Italy

\footnotetext{
*Speaker.

${ }^{\dagger}$ Great thanks to my mentor at GERDA/LNGS Carla Macolino, who taught me so well during my time in Italy
} 


\section{Introduction}

From September 22 to October 3 2014, the INFN (Istituto Nazionale di Fisica Nucleare) held the annual Gran Sasso Summer Institute at Laboratori Nazionali del Gran Sasso (LNGS) in Assergi, Italy. As part of the Institute, $\mathrm{PhD}$ students were given the opportunity to participate in hands-on work on an experiment of choice located in the Gran Sasso underground lab, under the supervision of a LNGS researcher. In the present paper, we report the results of the hands-on work within the GERDA experiment, for the search for neutrinoless double beta decay. The research activity consisted in the energy calibration and resolution measurement of a Broad Energy Germanium detector ${ }^{1}$ (BEGe) implemented in the Liquid Argon Germanium facility at LNGS.

\section{The GERDA experimental setup}

GERDA (GERmanium Detector Array) is an experiment meant to search for neutrinoless double beta decay $(0 v \beta \beta)$ in ${ }^{76} \mathrm{Ge}$; the observation of such decay would demonstrate the Majorana nature of neutrinos and violation of lepton number conservation. GERDA was originally proposed in 2004 and celebrated its inauguration with a completed setup in November 2010. In the first phase of the experiment a limit on the half-life of $0 v \beta \beta$ was established $\left(T_{1 / 2}^{0 v}>2.1 \cdot 10^{25} \mathrm{yr}\right.$ [3] $)$. Currently, GERDA is preparing for the second phase of its operation. In its first phase, GERDA operated High Purity Germanium (HPGe) and Broad Energy Germanium (BEGe) detectors, enriched in ${ }^{76} \mathrm{Ge}$ to about $86 \%$ and acting as both the $0 v \beta \beta$ decay source and a $4 \pi$ detector. The detectors were implemented inside a cryostat containing cryogenic liquid argon (LAr), surrounded by a further shield of ultra-pure water. In this way, the LAr can act both as coolant for the detectors and shield against external gamma radiation [1]. The Ge detectors are arranged in strings and suspended in the LAr in the center of the copper-lined, steel cryostat. Furthermore, a separate copper radon shroud is utilized to prevent radon from accumulating near the detectors due to convection. The cryostat assembly is placed in the water shield which provides gamma and neutron moderation. The water tank is instrumented with photomultiplier tubes to allow for detection of Cherenkov light acting as muon veto [2].

\section{The LArGe facility}

LArGe (Liquid Argon Germanium) is a GERDA low-background test facility which has been designed to study novel background suppression methods based on the scintillation light of LAr in a low-background environment for possible applications in the second phase of the GERDA experiment [4]. LArGe can operate up to $9 \mathrm{Ge}$ detectors in $1 \mathrm{~m}^{3}$ of LAr in a low-level environment (see Fig. 1). Unlike GERDA Phase I setup, the LAr cryostat is augmented with 9 photomultiplier tubes (PMTs), which can detect argon scintillation light. The cryostat is surrounded by a multilayered shield of increasing radiopurity in an attempt to suppress the external background. The outermost layer consists of $20 \mathrm{~cm}$ polyethylene, mainly to attenuate neutrons. This is followed by $23 \mathrm{~cm}$ of steel, $10 \mathrm{~cm}$ of low-activity lead, and $15 \mathrm{~cm}$ of electrolytic copper. Finally, the innermost layer consists of $41 \mathrm{~cm}$ of LAr, assuming a centrally positioned Ge-detector of $4 \mathrm{~cm}$ radius.

\footnotetext{
${ }^{1}$ manufactured by Canberra, Olen (Belgium).
} 


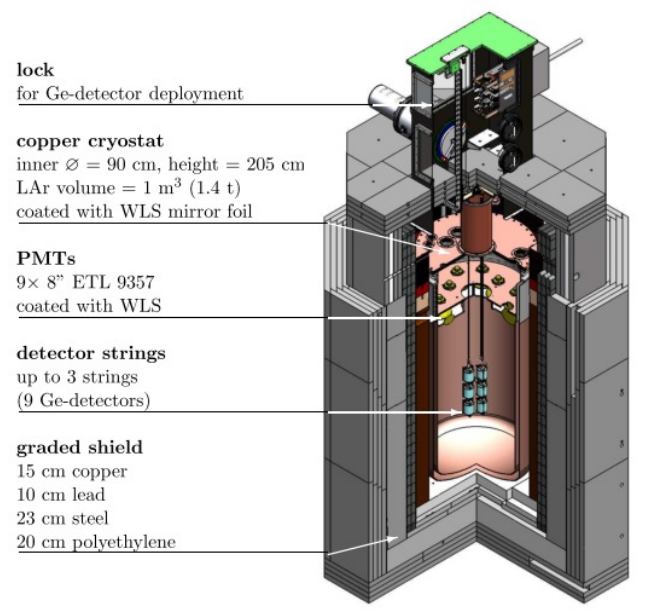

Figure 1: Cutaway view of the LArGe setup. The cryogenic infrastructure, a slow control system, and the DAQ are located adjacent to this setup. WLS = wavelength shifter [4].

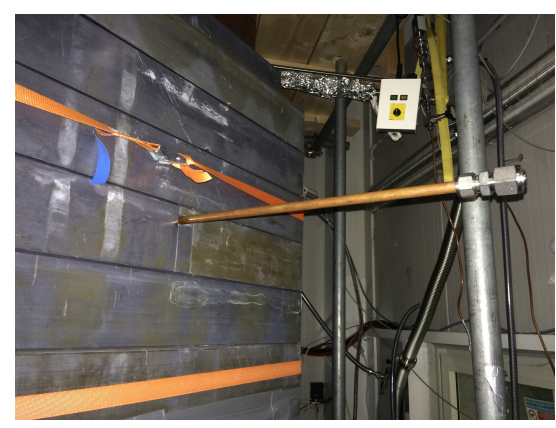

Figure 2: Source tube protruding from the LArGe detector.

Measurements performed in LArGe show that by detecting scintillation light originated in LAr from background components (and using it as a veto), a background level of about $10^{-3}$ cts/(keV·kg.y) can be reached at the Q-value of neutrinoless double beta decay of ${ }^{76} \mathrm{Ge}(2039$ $\mathrm{keV}$ ) [4]. Based on these results, GERDA Phase II will implement a LAr veto and, togheter with the addition of about $20 \mathrm{~kg}$ of BEGe detectors, it will be able to reach a sensitivity on half-life of neutrinoless double beta decay of $T_{1 / 2}^{0 v} \sim 1.4 \cdot 10^{26} \mathrm{yr}$ [5].

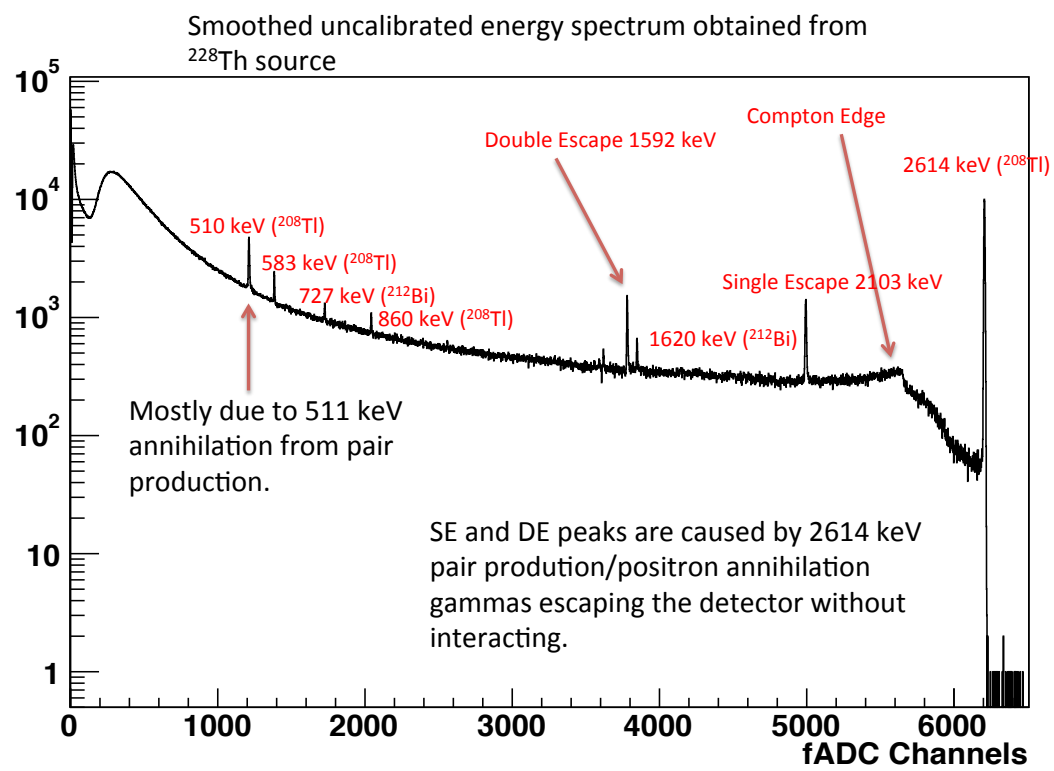

Figure 3: Uncalibrated spectrum from the ${ }^{228}$ Th source. 


\section{Calibration measurements}

In order to estimate the collected energy of the recorded events, GERDA detectors need to be calibrated. This is done by inserting a radioactive source of ${ }^{228} \mathrm{Th}$ and matching the detector's resultant output energy spectrum to the fADC channel response, through the identification of known energy peaks in the thorium spectrum. Also, this process allows to estimate the resolution of the

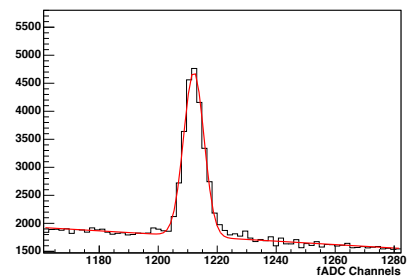

(a): The $510 \mathrm{keV}$ peak

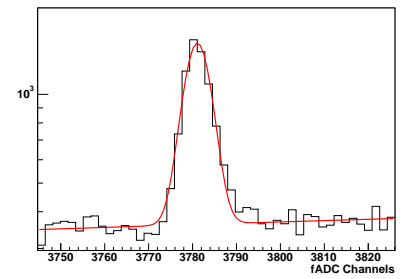

(d): The $1592 \mathrm{keV}$ double escape peak.

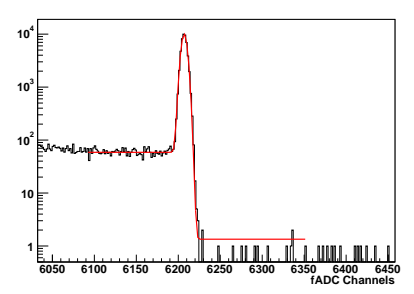

(g): The $2614 \mathrm{keV}$ peak.

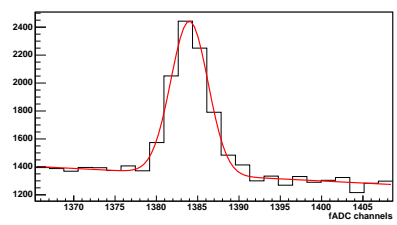

(b): The $583 \mathrm{keV}$ peak.

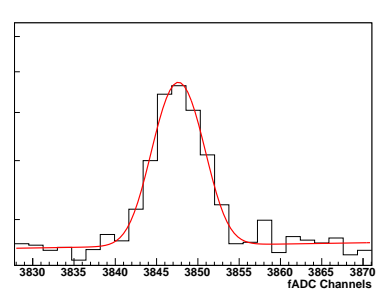

(e): The $1620 \mathrm{keV}$ peak.

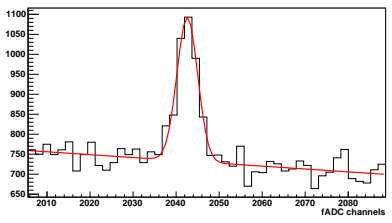

(c): The $860 \mathrm{keV}$ peak.

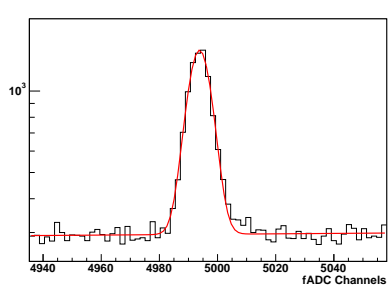

(f): The $2103 \mathrm{keV}$ peak.

Figure 4: Fitted peaks appearing in the recorded spectrum. The peaks were fitted by a Gaussian+linear function, with the exception of the $2614 \mathrm{keV}$ peak which was fitted with a constant+Gaussian+Step function+Tail function.

detector at all energies, specifically those near the region of interest (ROI) around the Q-value at $2039 \mathrm{keV}$. The first step is to insert the source and take spectrum data. The LArGe cryostat has been prepared with a tube allowing for easy placement of a source relatively near the detector crystal (see Fig. 2). A previously prepared ${ }^{228}$ Th source was inserted into the source tube and data taking proceeded over the weekend.

The data taken resulted in the production of an uncalibrated spectrum, shown in Fig. 3. Looking at this spectrum, we can easily recognize specific features: the photopeak at $2614 \mathrm{keV}$ from ${ }^{208} \mathrm{Tl}$, as well as the relative Compton Edge; also visible are the single and double escape peaks at $2103 \mathrm{keV}$ and $1592 \mathrm{keV}$, respectively. 


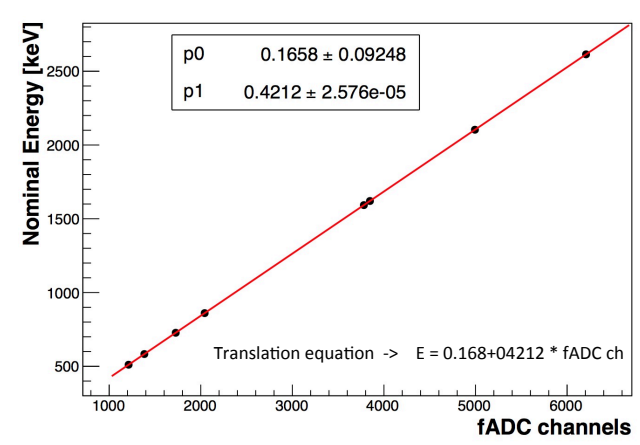

Figure 5: Calibration curve relating the nominal energies of the fitted peaks to the mean value of the fADC channels.

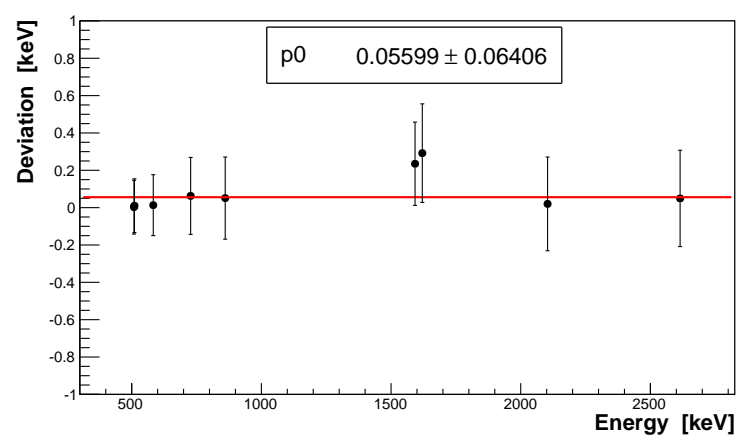

Figure 6: Relative deviation of the fitted energy values versus the nominal ones.

Once the spectrum has been obtained, the next step is to generate a fit function that will convert the fADC channel values into the absolute energies. This function is retrieved by performing a fit on several peaks and finding the corrisponding mean channel value. These mean channel values will be plotted with respect to the nominal energy of the gamma peak given in literature. Then, a curve fit will be performed on this plot and the resulting fit will determine the function that will be used to translate the energy spectrum. Using the ROOT software package, several peaks were examined to generate the mean channel value plot; the fitted peaks are illustrated in Fig. 4. The mean fADC channel was plotted versus the known energy and used to generate the fitted function. This is illustrated in Fig. 5. As expected, this relationship is linear; using this function, we can translate the measured spectrum to obtain a properly calibrated spectrum.

The translation equation we obtained is $E=0.168+0.4212 \times$ fADC channel (see Fig. 6). Applying this equation, the calibrated energy spectrum obtained is shown in Fig. 7.
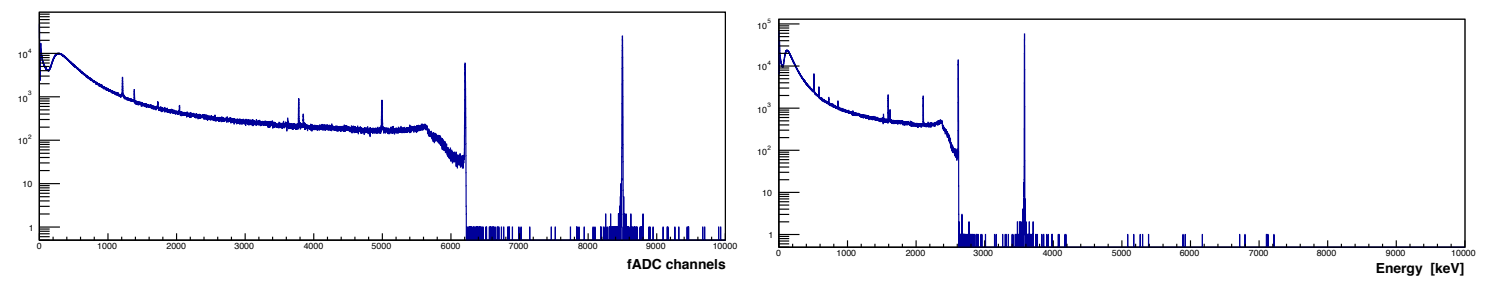

Figure 7: Uncalibrated recorded spectrum with Figure 8: Spectrum obtained by applying the the BEGe detector. calibration curve to the uncalibrated recorded spectrum.

\section{Energy resolution}

Energy resolution is a very important characteristic of gamma spectroscopic detectors in general, since high resolution (and the corresponding low peak width) enables to separate two gamma lines that are close to each other. Searching for neutrinoless double beta decay also requires the use of high energy resolution detectors, to allow a clear distinction of the $0 v \beta \beta$ peak from the detected 


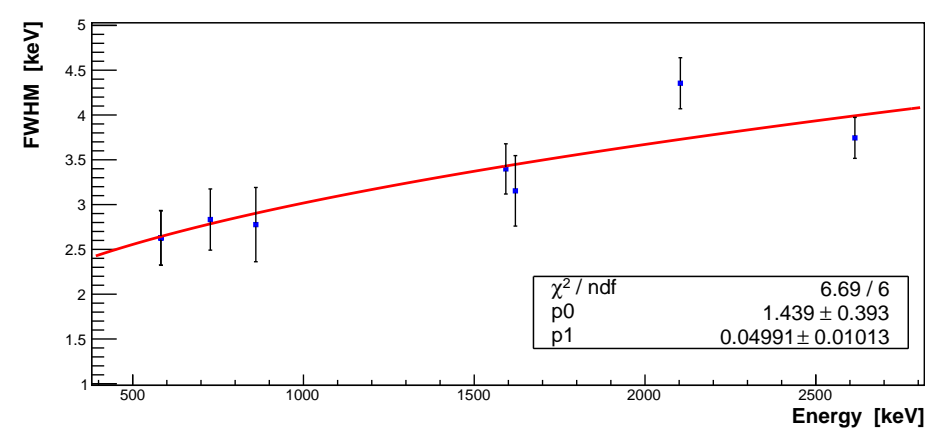

Figure 9: Full Width at Half Maximum versus energy for the different peaks, togheter with the resulting fitting function $\left(\mathrm{FWHM}=p_{0}+p_{1} \cdot \sqrt{E}\right)$.

spectrum.

To calculate the resolution of the BEGe detector considered, one must again examine the peaks at various energies, since this parameter is energy dependent. From the performed fit of each peak, one can estimate its standard deviation. The full width at half maximum (FWHM) can be obtained through the relation $\mathrm{FWHM}=2.355 \sigma$. By calculating the FWHM of the peaks seen in figure 4, one can generate a plot (shown in figure 9) showing the resolution across the entire useful energy range of the detector. Data can be fitted according to the function used in the Genie 2000 aquisition and analysis software provided by Canberra [6]: FWHM $=p_{0}+p_{1} \cdot \sqrt{E}$. According to the fit result, the estimated energy resolution (FWHM) of the detector at the Q-value is $3.7 \pm 0.8 \mathrm{keV}$. It is worth to mention that the estimation of the energy resolution is largely dependent on the working conditions in the laboratory, as for example the presence of people working near the setup, light switched on during the measurement, etc. We estimate a lower value of the energy resolution if the measurement was done in more quite conditions around the experimental setup.

\section{References}

[1] The GERDA collaboration, Eur. Phys. J., C, 73, 2330 (2013).

[2] C. Macolino for the GERDA collaboration, Mod. Phys. Lett. A 29, 20 (2013).

[3] The GERDA collaboration, Phys. Rev. Lett. 111, 12 (2013).

[4] M. Heisel, Ph.D. Dissertation, University of Heidelberg, Germany, (2011), Retrieved from http://pubman.mpdl.mpg.de/pubman/item/escidoc:1346653:3

[5] C. Macolino for the GERDA collaboration, J. Phys. Conf. Ser. 556, 012063 (2014).

[6] Web page: www.canberra.com. 